\title{
Ratiometric $\mathrm{pH}$ Sensing and Imaging in Living Cells with Dual-Emission Semiconductor Polymer Dots
}

\author{
Piaopiao Chen ${ }^{1}$, Iqra Ilyas ${ }^{1}$, Su He ${ }^{1}$, Yichen Xing ${ }^{1}$, Zhigang Jin ${ }^{1, * \mathbb{C}}$ and Chaobiao Huang ${ }^{1,2, *}$ \\ 1 College of Chemistry and Life Sciences, Zhejiang Normal University, Jinhua 321004, China \\ 2 Xingzhi College, Zhejiang Normal University, Jinhua 321004, China \\ * Correspondence: zgkin@zjnu.edu.cn (Z.J.); hcb@zjnu.cn (C.H.); Tel.: +86-579-8228-8003 (Z.J.); \\ Fax: +86-579-8228-2269 (Z.J.)
}

Received: 23 July 2019; Accepted: 10 August 2019; Published: 12 August 2019

\begin{abstract}
Polymer dots (Pdots) represent newly developed semiconductor polymer nanoparticles and exhibit excellent characteristics as fluorescent probes. To improve the sensitivity and biocompatibility of Pdots ratiometric $\mathrm{pH}$ biosensors, we synthesized 3 types of water-soluble Pdots: Pdots-PF, Pdots-PP, and Pdots-PPF by different combinations of fluorescent dyes poly(9,9-dioctylfluorenyl-2,7-diyl) (PFO), poly[(9,9-dioctyl-fluorenyl-2,7-diyl)-co-(1,4-benzo-\{2,1',3\}-thiadazole)] (PFBT), and fluorescein isothiocyanate (FITC). We found that Pdots-PPF exhibits optimal performance on $\mathrm{pH}$ sensing. $\mathrm{PFO}$ and FITC in Pdots-PPF produce $\mathrm{pH}$-insensitive $(\lambda=439 \mathrm{~nm})$ and $\mathrm{pH}$-sensitive $(\lambda=517 \mathrm{~nm})$ fluorescence respectively upon a single excitation at $380 \mathrm{~nm}$ wavelength, which enables Pdots-PPF ratiometric $\mathrm{pH}$ sensing ability. Förster resonance energy transfer (FRET) together with the use of PFBT amplify the FITC signal, which enables Pdots-PPF robust sensitivity to $\mathrm{pH}$. The emission intensity ratio $\left(\mathrm{I}_{517} / \mathrm{I}_{439}\right)$ of Pdots-PPF changes linearly as a function of $\mathrm{pH}$ within the range of $\mathrm{pH} 3.0$ to 8.0. Pdots-PPF also possesses desirable reversibility and stability in $\mathrm{pH}$ measurement. More importantly, Pdots-PPF was successfully used for cell imaging in Hela cells, exhibiting effective cellular uptake and low cytotoxicity. Our study suggests the promising potential of Pdots-PPF as an in vivo biomarker.
\end{abstract}

Keywords: Pdots; semiconductor polymer; FRET; pH sensing; cell imaging

\section{Introduction}

Intracellular $\mathrm{pH}$ is closely related to cellular behavior and pathological processes, including many types of cancer and neurodegenerative diseases [1]. Therefore, establishing and maintaining an appropriate $\mathrm{pH}$ in a single cell compartment is critical to its normal physiology. $\mathrm{pH}$ sensors have a wide range of applications such as food, beverages, environmental monitoring, chemical processing, biomedical applications, and laboratory $\mathrm{pH}$ measurements [2,3]. These applications typically require highly reliable and accurate $\mathrm{pH}$ sensors. Various techniques have been explored for $\mathrm{pH}$ measurement. Currently, the most common one is based on glass electrodes, which have also been recognized as commercial products with high stability and accuracy. However, they still have some drawbacks, such as the high brittleness of glass membrane electrodes, which create a strong incentive to develop new $\mathrm{pH}$ sensors $[4,5]$.

Fluorescent probes have proven to be an effective tool compared to other detection techniques such as electrochemical methods because of their many advantages, including simple operation, high sensitivity, selectivity, and in situ imaging [6,7]. To date, several materials have been used for $\mathrm{pH}$ sensing. For examples, Zhong et al. prepared carbon dots originated from carnation for fluorescent and colorimetric $\mathrm{pH}$ sensing [8]. Gao et al. designed a protease-activated ratiometric fluorescent probe based on Förster resonance energy transfer (FRET) between a pH-sensitive fluorescent dye and biocompatible $\mathrm{Fe}_{3} \mathrm{O}_{4}$ nanocrystals [9]. Kim et al. demonstrated a novel method for the preparation of a 
fluorescence-based $\mathrm{pH}$ sensor by combining the plasmon resonance band of $\mathrm{Ag}$ core and $\mathrm{pH}$ sensitive dye (HPTS) [10]. In addition, Ag metal mid layer, graphene, and other materials were also used for $\mathrm{pH}$ sensing [11-13]. However, these materials are limited by large size and high cytotoxicity in biological application. Fluorescent semiconductor Pdots have shown significantly improved behaviors and demonstrated as a suitable candidate for $\mathrm{pH}$ sensing. Recently, fluorescent semiconductor Pdots have attracted great interest. Semiconductor Pdots have proven to be unique features of fluorescence sensing and imaging, including high single particle brightness, excellent photostability, and low cytotoxicity, and have been developed for sensing, imaging, and photodynamic therapy [14-16].

Fluorescence sensors based on single wavelengths are easily disturbed by variable environmental conditions, so fluctuations in excitation sources and inaccurate probe concentrations have severely limited their application. In order to overcome these problems, the ratiometric fluorescence method was developed $[17,18]$. Fluorescence proportional sensing can effectively eliminate the deviation by measuring the fluorescence intensity ratio of two or more emission bands of different wavelengths, thereby improving the detection accuracy. Compared to the single fluorescence intensity-based fluorescence sensors, ratiometric fluorescent probes are rarely affected by excitation source fluctuations, probe concentration, probe photobleaching, instrumentation, or environmental influences [19-22].

In this study, we synthesized and optimized semiconductor Pdots via selective reprecipitation of two or three dyes: poly(9,9-dioctylfluorenyl-2,7-diyl) (PFO), poly[(9,9-dioctyl-fluorenyl-2,7-diyl)-co-(1,4-benzo- $\left\{2,1^{\prime}, 3\right\}$-thiadazole)] (PFBT), and fluorescein isothiocyanate (FITC) with cross-linking reagent poly(styrene-co-maleic anhydride) (PSMA). Comparison among these Pdots and previously reported FITC $\mathrm{pH}$ sensor confirmed that Pdots-PPF exhibited optimal performance on $\mathrm{pH}$ sensing. Pdots-PPF emitted dual fluorescence at the wavelength of $439 \mathrm{~nm}$ and $517 \mathrm{~nm}$ when excited at $380 \mathrm{~nm}$. The $\mathrm{pH}$-sensitive ratiometric Pdots-PPF provides a reliable method for monitoring $\mathrm{pH}$ based on the ratio of the variable fluorescence intensity at $517 \mathrm{~nm}$ to constant fluorescence intensity at $439 \mathrm{~nm}\left(\mathrm{I}_{517} / \mathrm{I}_{439}\right)$. We also showed that the synthesized Pdots-PPF possesses excellent biocompatibility and cell imaging property in Hela cells.

\section{Results and Discussion}

\subsection{Design of Ratiometric $p H$ Sensor Pdots-PF}

In order to improve currently available $\mathrm{pH}$-sensitive ratiometric Pdots for better performance on $\mathrm{pH}$ sensing and biocompatibility, we first chose $\mathrm{pH}$-insensitive dye $\mathrm{PFO}$ and $\mathrm{pH}$-sensitive dye FITC cross-linked by PMSA to prepare dual-emission semiconductor Pdots (Pdots-PF, Scheme 1). The emission spectra of PFO (peak at $439 \mathrm{~nm}$ and $465 \mathrm{~nm}$ ) and FITC (peak at $517 \mathrm{~nm}$ ) are obviously distinct (Figure 1a), which is a prerequisite for the design of dual emission ratiometric probes. We found the significant spectral overlap between the emission spectrum of PFO and the absorption spectrum of FITC, raising the possibility of FRET from PFO to FITC (Figure 1a). As depicted in Figure 1b, the synthesized Pdots-PF showed three emission bands upon excitation at $380 \mathrm{~nm}$. The intensity of Pdots-PF at $439 \mathrm{~nm}$ and $465 \mathrm{~nm}$ derived from PFO emission (blue line) was decreased as compared to that of PFO Pdots (black line), with concomitant increase of intensity at $517 \mathrm{~nm}$ derived from FITC emission (with same excitation at $380 \mathrm{~nm}$ ), indicating the occurrence of FRET from PFO to FITC.

The size of semiconductor polymer nanoparticles is important for cellular uptake and cytotoxicity during biological application. We utilized transmission electron microscopy (TEM) to determine the morphology of Pdots-PF. The TEM micrographs (Figure 1c) of Pdots-PF shows well dispersed nearly spherical particles, with an average diameter of $1.5 \pm 0.10 \mathrm{~nm}$ (Figure 1d). 


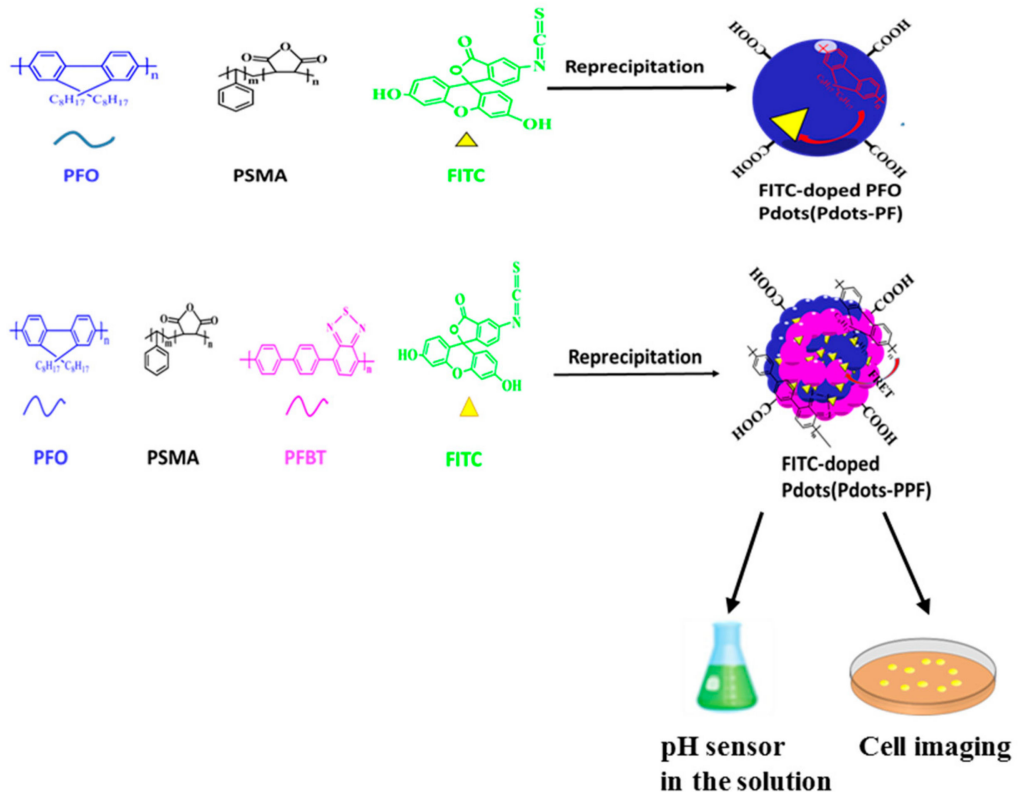

Scheme 1. A scheme showing the preparation and application of Pdots-PF and Pdots-PPF.

a
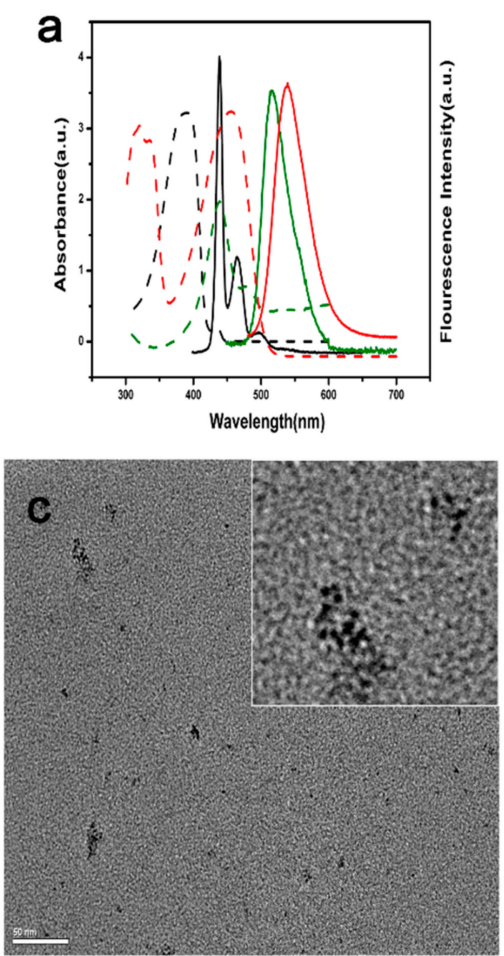
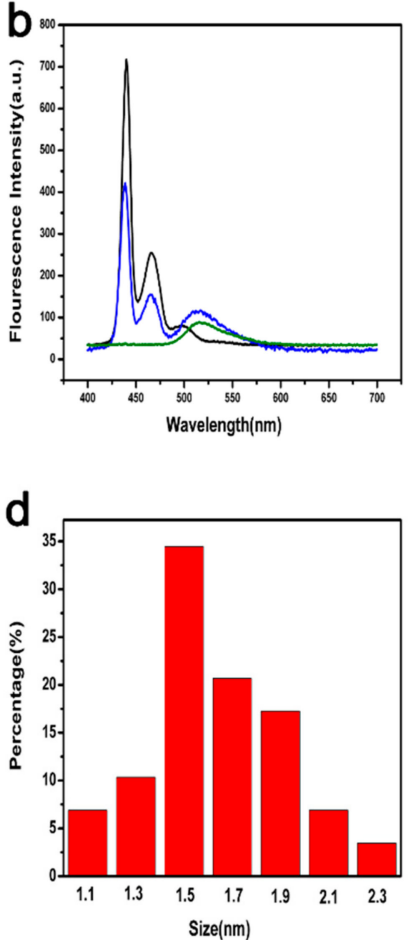

Figure 1. (a) Absorption of PFO (black dotted line), PFBT (red dotted line), and FITC (green dotted line), and emission spectra of PFO (black solid line), PFBT (red solid line), and FITC (green solid line) when excited at the optimal wavelength. (b) Emission spectra of PFO (black solid line), Pdots-PF (blue solid line), and FITC (green solid line) when excited at $380 \mathrm{~nm}$. (c) TEM of Pdots-PF. Scale bar represents $50 \mathrm{~nm}$. The inset shows the enlarged view of TEM of Pdots-PF. (d) The particle size distribution of Pdots-PF.

FITC has been applied as the pH sensor previously [23]. This was confirmed by our results (Figure 2a). However, as we mentioned earlier, the single fluorescent $\mathrm{pH}$ probes are easily disturbed by environment and call for ratiometric fluorescent probes. Next, we explored the dual-emission intensity changes of PFO Pdots and Pdots-PF in response to $\mathrm{pH}$. The fluorescence intensity of PFO Pdots hardly 
changes with $\mathrm{pH}$ (Figure $2 \mathrm{~b}$ ). With the increasing $\mathrm{pH}$ from 3.0 to 8.0, the fluorescence intensity of Pdots-PF showed different models: The emission peak of FITC (517 nm) increased while that of PFO (439 $\mathrm{nm}$ and $465 \mathrm{~nm}$ ) remained constant (Figure 2c). The invariable PFO emission suggested that both of the fluorescence intensity of PFO and the FRET efficiency are nonresponsive to $\mathrm{pH}$. Thus, we chose the fluorescence intensity of FITC $\left(\mathrm{I}_{517}\right)$ and PFO $\left(\mathrm{I}_{439}\right)$ as the response signal and reference signal, respectively. As shown in inset of Figure $2 \mathrm{c}$, we characterized the emission ratio $\mathrm{I}_{517} / \mathrm{I}_{439}$ of Pdots-PF. However, the linear relationship of $\mathrm{I}_{517} / \mathrm{I}_{439}$ to $\mathrm{pH}$ is not ideal in the range of $\mathrm{pH} 3.0$ to 8.0 , and the correlation coefficient is 0.9785 . We speculate that this is probably caused by the relatively weak signal of FITC upon excitation at $380 \mathrm{~nm}$, thus the $\mathrm{pH}$-dependent change of FITC intensity is subtle.
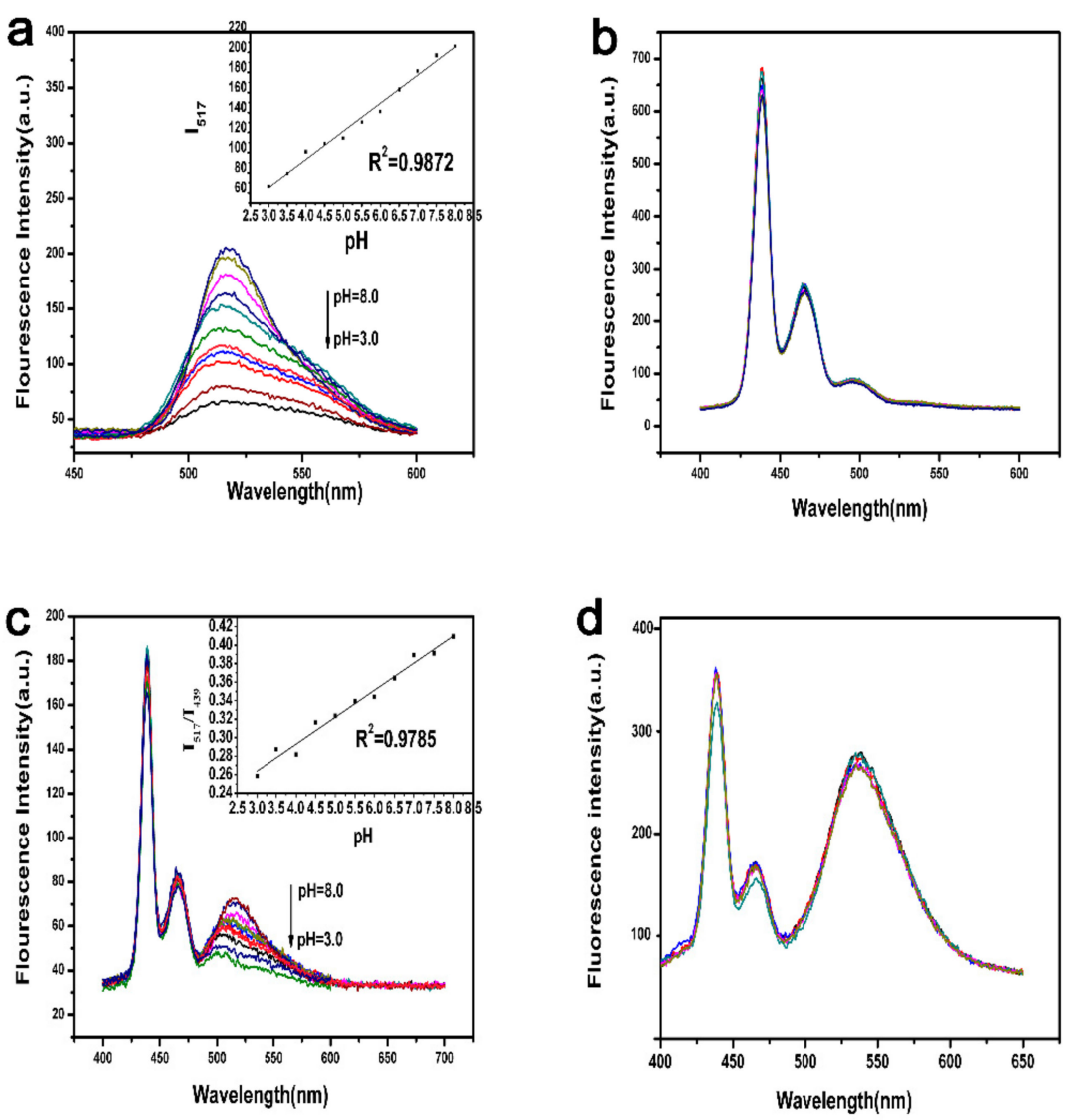

Figure 2. (a) Fluorescence emission spectra of FITC in pH 3.0-8.0 when excited at $488 \mathrm{~nm}$, inset shows the linear correlation between the $\mathrm{I}_{517}$ and the $\mathrm{pH}$. (b) Fluorescence emission spectra of PFO Pdots in $\mathrm{pH} 3.0-8.0$ when excited at $380 \mathrm{~nm}$. (c) Fluorescence emission spectra of Pdots-PF in pH 3.0-8.0 when excited at $380 \mathrm{~nm}$, inset shows the linear correlation between the $\mathrm{I}_{517} / \mathrm{I}_{439}$ and the $\mathrm{pH}$. (d) Fluorescence emission spectra of Pdots-PP in pH 3.0-8.0 when excited at $380 \mathrm{~nm}$.

\subsection{Design of Ratiometric $p H$ Sensor Pdots-PPF}

In order to address the above issue and improve the performance of Pdots-PF on $\mathrm{pH}$ sensing, we try to introduce PFBT, a polymer dye with similar emission wavelength of FITC (Figure 1a), to amplify the FITC signal and prepare Pdots-PPF, which contains fluorescent dyes PFO, PFBT, FITC, and cross-linking reagent PSMA.

Pdots-PPF was synthesized according to Scheme 1. As comparison, we also prepared Pdots-PP, which contains PFO, PFBT, and PSMA. The spectral overlap between the emission spectrum of PFO and the absorption spectrum of PFBT indicates the potential FRET from PFO to PFBT (Figure 1a). We examined the $\mathrm{pH}$ response of Pdots-PP and confirmed that Pdots-PP is $\mathrm{pH}$ nonresponsive in the range of $\mathrm{pH} 3.0$ to 8.0 (Figure 2d). We compared the fluorescence spectra of Pdots-PP and Pdots-PPF 
(Figure 3a), and found that the addition of FITC to Pdots-PP caused: (1) A blue-shift from $539 \mathrm{~nm}$ to $517 \mathrm{~nm}$. Therefore, the emission peaks of PFBT and FITC were merged at $517 \mathrm{~nm}$ and FITC signal was successfully amplified in Pdots-PPF. (2) A decreased fluorescence intensity of PFO at $439 \mathrm{~nm}$ and $465 \mathrm{~nm}$, indicating that similar to Pdots-PF, Pdots-PPF also showed efficient FRET from PFO to FITC/PFBT.
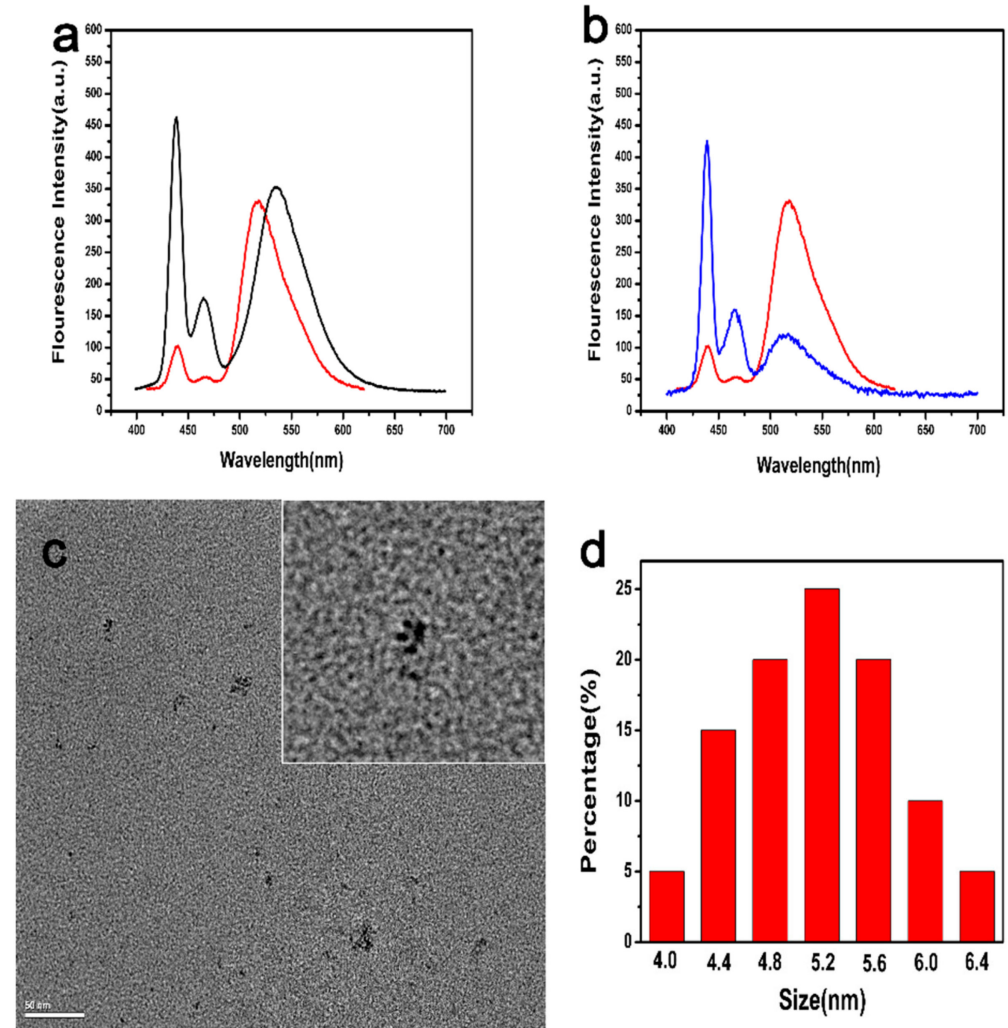

Figure 3. (a) Fluorescence spectra of Pdots-PP (black solid line) and Pdots-PPF (red solid line) when excited at $380 \mathrm{~nm}$. (b) Fluorescence spectra of Pdots-PF (blue solid line) and Pdots-PPF (red solid line) when excited at $380 \mathrm{~nm}$. (c) TEM of Pdots-PPF. Scale bar represents $50 \mathrm{~nm}$. The inset shows the enlarged view of TEM of Pdots-PPF. (d) The particle size distribution of Pdots-PPF.

Comparison of fluorescence spectra of Pdots-PF and Pdots-PPF confirmed that the weak FITC signal in Pdots-PF is replaced by a merged and amplified FITC/PFBT signal in Pdots-PPF (Figure 3b). The TEM images show that Pdots-PPF exhibits highly spherical monodispersity with a diameter of approximately $5.2 \pm 0.20 \mathrm{~nm}$ (Figure 3c,d). We summarized the composition of Pdots-PPF and the roles of each component in Table 1, and the comparison of $\mathrm{pH}$ sensors used in this study in Table 2.

Table 1. The composition of Pdots-PPF and the roles of each component.

\begin{tabular}{cc}
\hline Components & Functions \\
\hline PFO & FRET donor, provide reference signal $\left(\mathrm{I}_{439}\right)$ \\
\hline PFBT & FRET acceptor, FITC signal amplifier \\
\hline FITC & FRET acceptor, provide pH-responsive signal $\left(\mathrm{I}_{517}\right)$ \\
\hline PMSA & Cross-linking \\
\hline
\end{tabular}


Table 2. Comparison of $\mathrm{pH}$ sensors used in this study.

\begin{tabular}{ccccc}
\hline pH Sensors & Components & FRET & Emission & pH Sensitivity \\
\hline FITC & FITC & No & Single & Robust \\
\hline PFO Pdots & PFO, PMSA & No & Single & No \\
\hline Pdots-PF & PFO, FITC, PMSA & PFO to FITC & Dual & Weak \\
\hline Pdots-PP & PFO, PFBT, PMSA & PFO to PFBT & Dual & No \\
\hline Pdots-PPF & PFO, PFBT, FITC, PMSA & PFO to FITC/PFBT & Dual & Robust \\
\hline
\end{tabular}

\subsection{Ratiometric Fluorescence Detection of pH in Buffer Solution}

We speculated that the merged FITC/PFBT signal remains $\mathrm{pH}$-sensitive and PFO signal remains $\mathrm{pH}$-insensitive in Pdots-PPF. To test this possibility, the $\mathrm{pH}$ sensitivity and ratiometric property of Pdots-PPF were examined in HEPES buffer solutions with various $\mathrm{pH}$ values. As we expected, the fluorescence intensity of Pdots-PPF at $517 \mathrm{~nm}$ increased when the $\mathrm{pH}$ was increased from 3.0 to 8.0 and fluorescence intensity at $439 \mathrm{~nm}$ is relatively constant (Figure 4a). Therefore, the $\mathrm{pH}$-dependent change of FITC intensity is significantly improved in Pdots-PPF ( $\mathrm{pH} 8.0$ vs. $\mathrm{pH}$ 3.0, fold change of $\mathrm{I}_{517}$ is 2.77 ) as compared to Pdots-PF (pH 8.0 vs. $\mathrm{pH} 3.0$, fold change of $\mathrm{I}_{517}$ is 1.66).
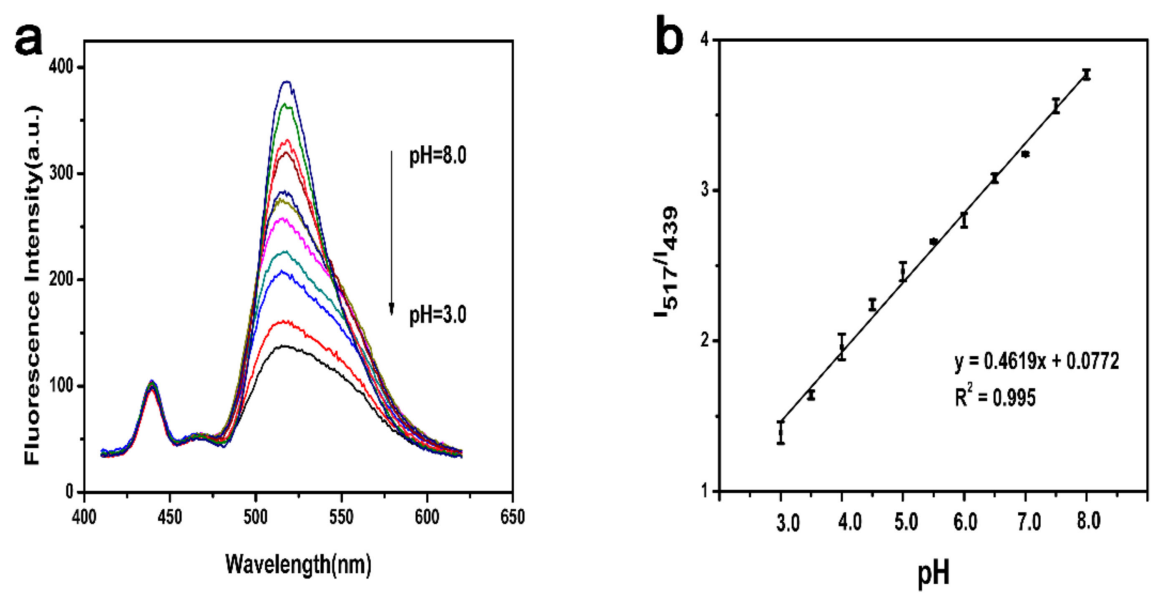

Figure 4. (a) Fluorescence emission spectra of Pdots-PPF at pH 3.0-8.0 in 20.0 mM HEPES buffer solution when excited at $380 \mathrm{~nm}$. (b) Plot of the relative fluorescence intensity ratios $\left(\mathrm{I}_{517} / \mathrm{I}_{439}\right)$ of Pdots-PPF in response to $\mathrm{pH}$ values.

Next, we examined the relationship between the ratio of the emission intensities of FITC $\left(\mathrm{I}_{517}\right)$ to $\mathrm{PFO}\left(\mathrm{I}_{439}\right)$ and the $\mathrm{pH}$ values (Figure $4 \mathrm{~b}$ ). The change in fluorescence ratio of $\mathrm{I}_{517} / \mathrm{I}_{439}$ shows a good linear relationship with the $\mathrm{pH}$ values. The linear equation is $\mathrm{y}=0.6149 \mathrm{x}+0.0772$, and the correlation coefficient is 0.995 . However, the linear relationship is lost when the $\mathrm{pH}$ values are beyond the range of $\mathrm{pH} 3.0$ to 8.0 (data not shown). We conclude that Pdots-PPF exhibits significant advantage over Pdots-PF and FITC as a ratiometric $\mathrm{pH}$ sensor and can be applied for $\mathrm{pH}$ detection within the linear range between $\mathrm{pH} 3.0$ and 8.0 (Table 2).

\subsection{Reversibility, Stability, and Selectivity of the $\mathrm{pH}$ Sensors}

The reversibility of Pdots-PPF was examined by cyclic detection of $\mathrm{pH}$ at $\mathrm{pH} 3.0$ and 8.0 (Figure 5a). Pdots-PPF shows excellent reversibility between $\mathrm{pH} 3.0$ and 8.0, which is attributed to the inherent characteristic of the fast protonation/deprotonation process of FITC and the desirable stability of the fluorescent probe. 

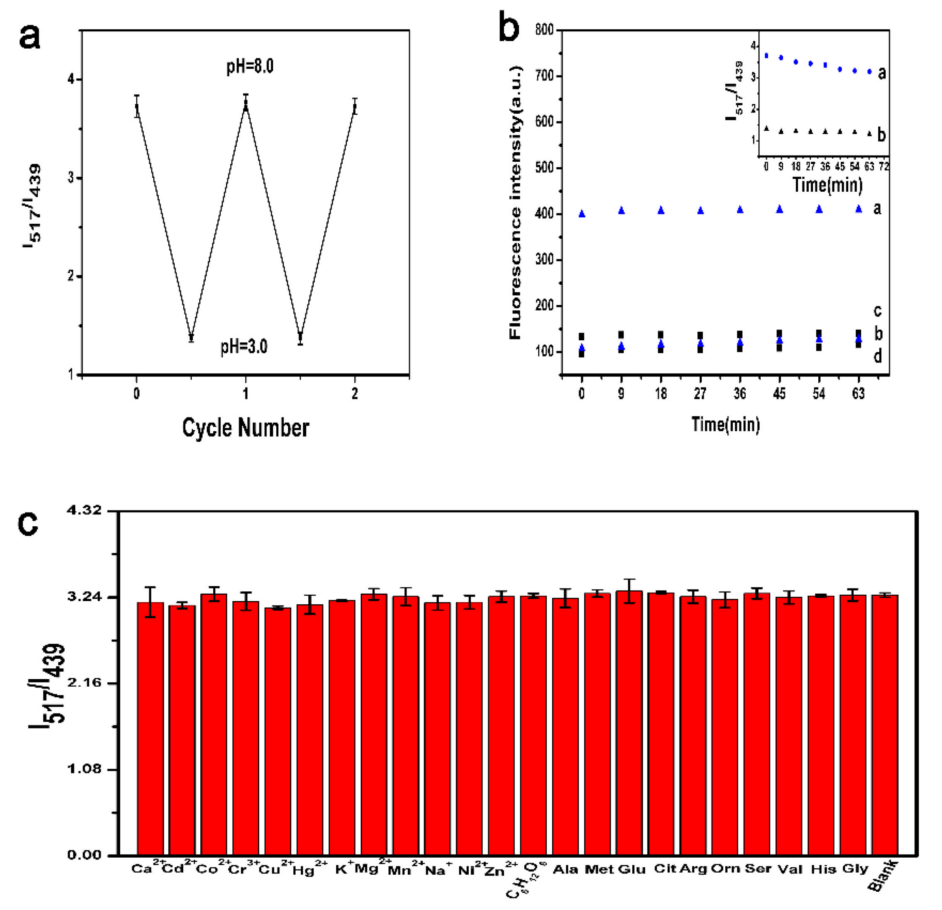

Figure 5. (a) Fluorescence intensity ratios $\left(\mathrm{I}_{517} / \mathrm{I}_{439}\right)$ of Pdots-PPF upon repeatedly switching $\mathrm{pH}$ from 3.0 to 8.0. (b) Fluorescence intensity of $\lambda_{517}(a, c)$ and $\lambda_{439}(b, d)$ emission of Pdots-PPF in $20.0 \mathrm{mM}$ HEPES buffer solution at $\mathrm{pH} 8.0$ and 3.0. The inset: The corresponding time-dependent intensity ratios $\left(\mathrm{I}_{517} / \mathrm{I}_{439}\right)$ of Pdots-PPF at $\mathrm{pH}=8.0$ (a) and 3.0 (b). (c) Selectivity of the Pdots-PPF.

Furthermore, the photostability of Pdots-PPF at different $\mathrm{pH}$ values were investigated. Figure $5 \mathrm{~b}$ shows the individual time-dependent fluorescence intensity of the emission at $517 \mathrm{~nm}(\mathrm{a}, \mathrm{c})$ and $439 \mathrm{~nm}$ $(\mathrm{b}, \mathrm{d})$ of Pdots-PPF at different $\mathrm{pH}$ values under $365 \mathrm{~nm}$ UV lamp illumination. In summary, both the individual fluorescence intensity and the ratio of Pdots-PPF in different $\mathrm{pH}$ buffers (Figure $5 \mathrm{~b}$ inset) did not show significant changes with duration of $63 \mathrm{~min}$, indicating that Pdots-PPF exhibited outstanding photostability and could be used for long term monitoring of $\mathrm{pH}$.

Selectivity is an important aspect in the application of $\mathrm{pH}$ sensors. In order to investigate the anti-interfering ability of Pdots-PPF for $\mathrm{pH}$ sensing, the variations in fluorescence intensity ratios $\left(\mathrm{I}_{517} / \mathrm{I}_{439}\right)$ in the presence of potential interfering substances including ions and amino acids were examined under the same conditions. As depicted in Figure $5 c$, compared to the blank $\mathrm{I}_{517} / \mathrm{I}_{439}$ ratio in the absence of interfering substances, no detectable variations in the $\mathrm{I}_{517} / \mathrm{I}_{439}$ ratios were observed while adding different interfering species such as $100 \mu \mathrm{M}$ of $\mathrm{Ca}^{2+}, \mathrm{Cd}^{2+}, \mathrm{Co}^{2+}, \mathrm{Cr}^{3+}, \mathrm{Cu}^{2+}, \mathrm{Hg}^{2+}, \mathrm{K}^{+}$, $\mathrm{Mg}^{2+}, \mathrm{Mn}^{2+}, \mathrm{Na}^{+}, \mathrm{Ni}^{2+}, \mathrm{Zn}^{2+}$, and $10 \mu \mathrm{M}$ of glucose, alanine(Ala), methionine (Met), glutamic acid (Glu), citrulline(Cit), L-arginine (Arg), ornithine (Orn), serine (Ser), valine (Val), histidine (His), glycine (Gly) in HEPES (20 mM pH 7.0). The anti-interfering experiment reveals that Pdots-PPF displays the capacity for $\mathrm{pH}$ determination with high selectivity.

\subsection{Cell Imaging Applications of Pdots-PPF}

The cell viabilities were examined upon exposure to the Pdots-PPF of different concentrations. Figure 6 illustrates that the Pdots-PPF exhibited extremely low cytotoxicity, with cell viability over 93\% at the concentration of $0.3,0.5,1.5,2.5,5.0 \mu \mathrm{g} \cdot \mathrm{mL}^{-1}$ Pdots-PPF after incubation for $24 \mathrm{~h}$. 


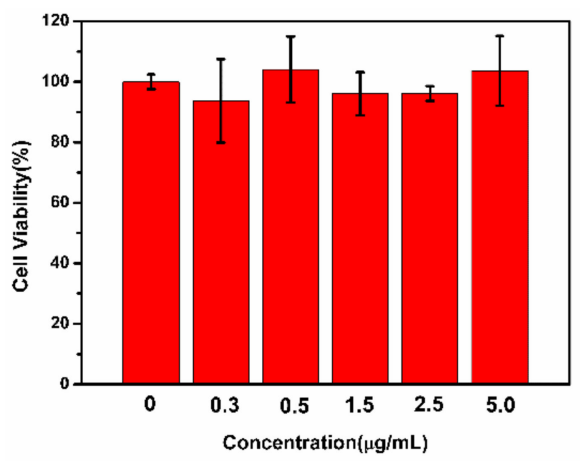

Figure 6. MTT based cytotoxicity assay of Hela cells after $24 \mathrm{~h}$ incubation with different concentrations of Pdots-PPF $\left(0,0.3,0.5,1.5,2.5\right.$, and $\left.5.0 \mu \mathrm{g} \cdot \mathrm{mL}^{-1}\right)($ mean $\pm \mathrm{SD}, \mathrm{n}=3)$.

In order to investigate the potential of Pdots-PPF for intracellular $\mathrm{pH}$ measurements, we incubated Pdots-PPF with live Hela cells to allow cellular uptake via endocytosis. After cellular uptake, we washed Hela cells to remove extracellular Pdots-PPF. The bright-field and fluorescent images of Hela cells with intracellular Pdots-PPF are depicted in Figure 7. The Hela cells labeled with Pdots-PPF exhibited bright blue and green fluorescence under ultraviolet (340-380 nm) and blue (450-490 nm) light excitation, respectively. Close observations revealed that the Pdots-PPF was distributed mainly within the nucleus, while cell membrane and the cytoplasmic area had only weak fluorescence.

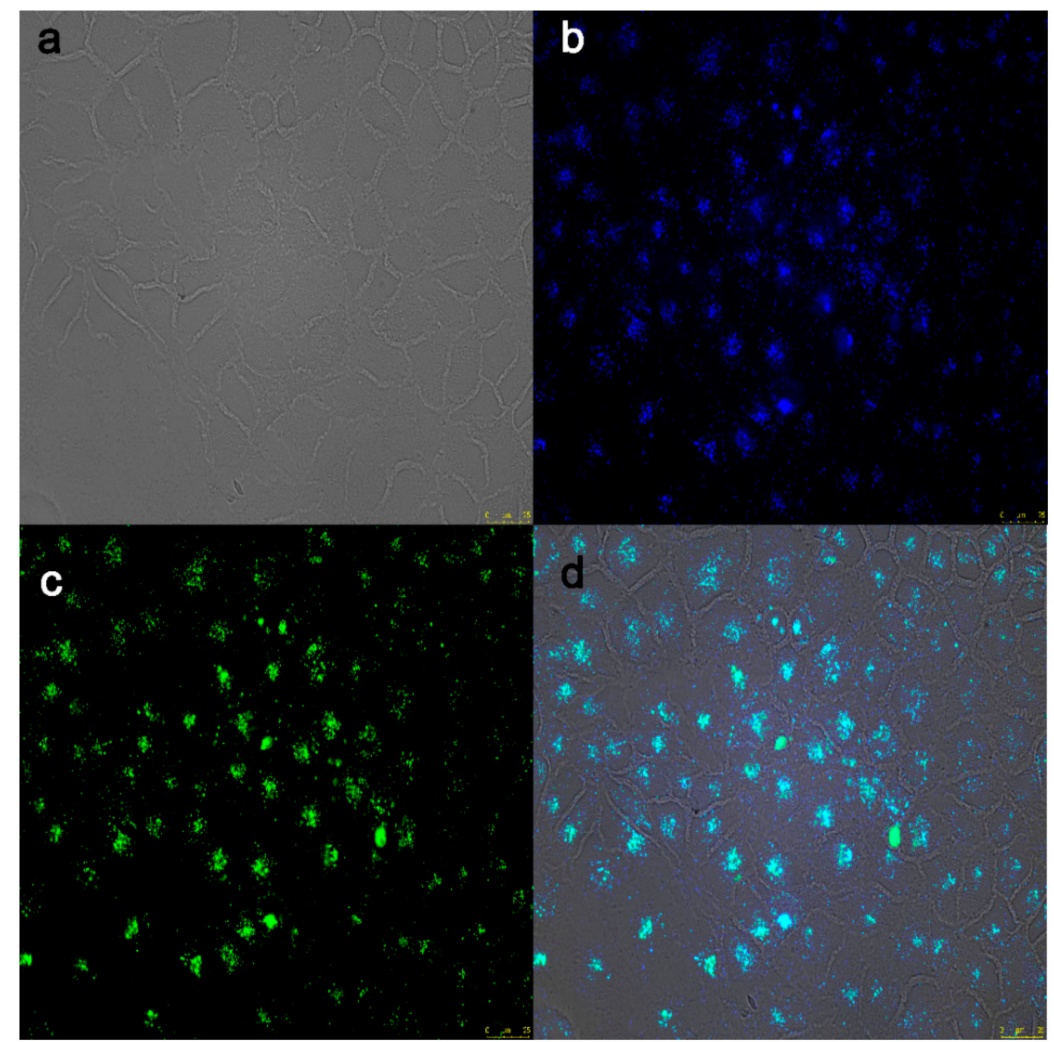

Figure 7. (a) Bright-field and (a-c) fluorescent images of Hela cells incubated with Pdots-PPF. (d) merged image of (a), (b) and (c). Scale bar represents $25 \mu \mathrm{m}$.

The above experiments indicate that the Pdots-PPF is featured with photostabilities, high resistance to photobleaching, inertness to interference of metal ions and biomolecular species, and excellent biocompatibility, suggesting Pdots-PPF to be a promising candidate for bioimaging and $\mathrm{pH}$ biosensor applications under physiological or pathological conditions. 


\section{Experimental Section}

\subsection{Materials and Reagents}

Alanine (Ala), methionine (Met), glutamic acid (Glu), citrulline (Cit), L -arginine (Arg), ornithine(Orn), serine(Ser), valine(Val), histidine(His), glycine (Gly), 2-(4-(2-hydroxyethyl)1-piper-azinyl) ethanesulfonic acid buffer (HEPES,) and fluorescein isothiocyanate (FITC) were purchased from Aladdin (Shanghai, China). Poly(9,9-dioctylfluorenyl-2,7-diyl) (PFO), Poly[(9,9-dioctyl-fluorenyl-2,7-diyl)-co-(1,4-benzo- $\left\{2,1^{\prime}, 3\right\}$-thiadazole) $\quad$ (PFBT) and poly(styrene-co-maleic anhydride) (PSMA) were purchased from Sigma-Aldrich (MO, USA). Tetrahydrofuran (THF), glucose, $\mathrm{NaCl}, \mathrm{KCl}, \mathrm{CoCl}_{2} \cdot 6 \mathrm{H}_{2} \mathrm{O}, \mathrm{CaCl}_{2} \cdot 2 \mathrm{H}_{2} \mathrm{O}, \mathrm{MnCl}_{2} \cdot 4 \mathrm{H}_{2} \mathrm{O}, \mathrm{CdCl}_{2} \cdot 2.5 \mathrm{H}_{2} \mathrm{O}$, $\mathrm{ZnCl}_{2}, \mathrm{NiCl}_{2} \cdot 6 \mathrm{H}_{2} \mathrm{O}, \mathrm{CuCl}_{2} \cdot 2 \mathrm{H}_{2} \mathrm{O}, \mathrm{MgCl}_{2} \cdot 6 \mathrm{H}_{2} \mathrm{O}, \mathrm{CrCl}_{3} \cdot 6 \mathrm{H}_{2} \mathrm{O}$, and $\mathrm{Hg}\left(\mathrm{NO}_{3}\right)_{2}$ were purchased from Dongnan Chemical Reagent (Zhejiang, China). All reagents were used as received without further purification.

\subsection{Instruments}

The morphology of Pdots was characterized by transmission electron microscopy (TEM) using a JEM-2100F TEM facility. The UV-vis absorption spectrum was obtained with a LZMBDA 950 spectrophotometer. Fluorescence (FL) spectra were recorded using a RF-5301PC fluorescence spectrophotometer (Shimadzu, Japan). Fluorescence were observed and imaged on a Leica DM4B fluorescence microscope. Absorbance of samples for cytotoxicity assay was measured by microplate reader (Bio-Rad iMark Microplate Absorbance Reader, Hercules, CA, USA).

\subsection{Preparation of Pdots-PF, Pdots-PP, and Pdots-PPF}

Pdots were synthesized according to a reported method [22]. FITC and polymers PFO, PFBT, and PSMA were dissolved in anhydrous THF to obtain $1.0 \mathrm{mg} \cdot \mathrm{mL}^{-1}$ stock solutions under sonication, respectively.

To prepare Pdots-PF, the solutions of PFO, PSMA, and FITC in THF were mixed to make a solution mixture with PFO concentration of $30 \mu \mathrm{g} \cdot \mathrm{mL}^{-1}$, PSMA concentration of $16 \mu \mathrm{g} \cdot \mathrm{mL}^{-1}$, and FITC concentration of $60 \mu \mathrm{g} \cdot \mathrm{mL}^{-1}$. A $3 \mathrm{~mL}$ quantity of the solution mixture was quickly injected into $10 \mathrm{~mL}$ of ultrapure water and sonicated for $5 \mathrm{~min}$, and then THF was removed by reduced pressure distillation to get a colloidal solution of Pdots-PF. After that, the as-prepared colloidal solution was filtered through a $0.22 \mu \mathrm{m}$ membrane filter to remove larger aggregates. The prepared Pdots-PF solution was stored at $4{ }^{\circ} \mathrm{C}$.

To prepare Pdots-PP, the solutions of PFO, PFBT, and PSMA were mixed to make a solution mixture with PFO concentration of $30 \mu \mathrm{g} \cdot \mathrm{mL}^{-1}$, PFBT concentration of $20 \mu \mathrm{g} \cdot \mathrm{mL}^{-1}$, and PSMA concentration of $16 \mu \mathrm{g} \cdot \mathrm{mL}^{-1}$. The subsequent processing is the same as Pdots-PF. The Pdots-PP concentration was $15 \mu \mathrm{g} \cdot \mathrm{mL}^{-1}$ and then the prepared Pdots-PP solution was stored at $4{ }^{\circ} \mathrm{C}$.

To prepare Pdots-PPF, the solutions of PFO, PFBT, PSMA, and FITC in THF were mixed to make a solution mixture with PFO concentration of $30 \mu \mathrm{g} \cdot \mathrm{mL}^{-1}$, PFBT concentration of $20 \mu \mathrm{g} \cdot \mathrm{mL}^{-1}$, PSMA concentration of $16 \mu \mathrm{g} \cdot \mathrm{mL}^{-1}$, and FITC concentration of $60 \mu \mathrm{g} \cdot \mathrm{mL}^{-1}$. The subsequent processing is the same as Pdots-PF. The Pdots-PPF concentration was $15 \mu \mathrm{g} \cdot \mathrm{mL}^{-1}$ and then the prepared Pdots-PPF solution was stored at $4{ }^{\circ} \mathrm{C}$.

\subsection{Procedures for $\mathrm{pH}$ Detection}

An equivalent amount of $20 \mu \mathrm{L}$ of Pdots $\left(15 \mu \mathrm{g} \cdot \mathrm{mL}^{-1}\right)$ solution was put into a series of $2 \mathrm{~mL}$ centrifuge tubes and diluted to $1 \mathrm{~mL}$ using HEPES buffers $(20 \mathrm{mM})$ with various $\mathrm{pH}$ values, respectively. The fluorescence measurements were performed with $380 \mathrm{~nm}$ light excitation for Pdots and $488 \mathrm{~nm}$ light excitation for FITC. 


\subsection{Cell Culture and Cell Imaging of Pdots}

Hela cells (CCL-2, epithelial of human cervical cancer cells, ATCC, Manassas, VA, United States) were maintained in DMEM supplemented with $10 \%$ fetal bovine serum, penicillin, and streptomycin, at $37{ }^{\circ} \mathrm{C}$ in a humidified, $5 \% \mathrm{CO}_{2}$ atmosphere. Hela cells were treated with $3.0 \mu \mathrm{g} \cdot \mathrm{mL}^{-1}$ Pdots. After incubation for $24 \mathrm{~h}$ in the dark, Hela cells were washed with PBS buffer for 3 times and harvested for cell imaging with Leica DM4B fluorescence microscope and the LAS X software (Leica Microsystems, Wetzlar, Germany).

\subsection{Cytotoxicity Assay}

Hela cells were treated with indicated concentrations of Pdots for $24 \mathrm{~h}$. Ten (10) $\mu \mathrm{L}$ of 3-(4,5-dimethythiazol-2-yl)-2,5-diphenyl tetrazolium bromide (MTT) was added. Cells were incubated at $37^{\circ} \mathrm{C}$ for $4 \mathrm{~h}$ and then lysed by $100 \mu \mathrm{L}$ of lysis buffer ( $4 \%$ Triton $\mathrm{X}-100$ and $0.14 \% \mathrm{HCl}$ in 2-propanol). Absorbance at $595 \mathrm{~nm}$ was measured on a microplate reader (Bio-Rad, Hercules, CA, USA).

\section{Conclusions}

In general, we optimized semiconductor Pdots and obtained Pdots-PPF with optimal behavior for $\mathrm{pH}$ detection. Each component of Pdots-PPF is indispensable and plays an important role in ratiometric pH sensing. Ultimately, Pdots-PPF can be successfully applied for cell imaging, indicating its promising potential for in vivo bioimaging and biosensing, which is currently under our investigation.

Author Contributions: Conceptualization, P.C., Z.J. and C.H.; methodology, P.C., I.I., S.H. and Y.X.; data curation, P.C., I.I., S.H. and Y.X.; formal analysis, P.C. and Z.J.; writing-original draft, P.C.; writing-review and editing, Z.J. and C.H. All authors read and approved the final manuscript.

Funding: The authors gratefully acknowledge the support for this research by the National Natural Science Foundation of China (21575129).

Conflicts of Interest: The authors have no conflicts of interest to declare.

\section{References}

1. Shi, W.; Li, X.; Ma, H. A tunable ratiometric $\mathrm{pH}$ sensor based on carbon nanodots for the quantitative measurement of the intracellular pH of whole cells. Angew. Chem. Int. Ed. 2012, 51, 6432-6435. [CrossRef] [PubMed]

2. Näreoja, T.; Deguchi, T.; Christ, S.; Peltomaa, R.; Prabhakar, N.; Fazeli, E.; Perälä, N.; Rosenholm, J.M.; Arppe, R.; Soukka, T. Ratiometric sensing and imaging of intracellular $\mathrm{pH}$ using polyethylenimine-coated photon upconversion nanoprobes. Anal. Chem. 2017, 89, 1501-1508. [CrossRef] [PubMed]

3. Consolati, T.; Bolivar, J.M.; Petrasek, Z.; Berenguer, J.; Hidalgo, A.; Guisaán, J.M.; Nidetzky, B. Biobased, Internally $\mathrm{pH}$-Sensitive Materials: Immobilized Yellow Fluorescent Protein as an Optical Sensor for Spatiotemporal Mapping of pH Inside Porous Matrices. ACS Appl. Mater. Interfaces 2018, 10, 6858-6868.

4. Ratanaporncharoen, C.; Tabata, M.; Kitasako, Y.; Ikeda, M.; Goda, T.; Matsumoto, A.; Tagami, J.; Miyahara, Y. $\mathrm{pH}$ Mapping on Tooth Surfaces for Quantitative Caries Diagnosis Using Micro Ir/IrOx pH Sensor. Anal. Chem. 2018, 90, 4925-4931. [CrossRef] [PubMed]

5. Zheng, Y.-B.; Zhao, S.; Cao, S.-H.; Cai, S.-L.; Cai, X.-H.; Li, Y.-Q. A temperature, pH and sugar triple-stimuli-responsive nanofluidic diode. Nanoscale 2017, 9, 433-439. [CrossRef] [PubMed]

6. Zhao, S.; Lan, M.; Zhu, X.; Xue, H.; Ng, T.-W.; Meng, X.; Lee, C.-S.; Wang, P.; Zhang, W. Green synthesis of bifunctional fluorescent carbon dots from garlic for cellular imaging and free radical scavenging. ACS Appl. Mater. Interfaces 2015, 7, 17054-17060. [CrossRef] [PubMed]

7. Li, S.; Chang, K.; Sun, K.; Tang, Y.; Cui, N.; Wang, Y.; Qin, W.; Xu, H.; Wu, C. Amplified singlet oxygen generation in semiconductor polymer dots for photodynamic cancer therapy. ACS Appl. Mater. Interfaces 2015, 8, 3624-3634.

8. Zhong, D.; Miao, H.; Yang, K.; Yang, X. Carbon dots originated from carnation for fluorescent and colorimetric pH sensing. Mater. Lett. 2016, 166, 89-92. [CrossRef] 
9. Hou, Y.; Zhou, J.; Gao, Z.; Sun, X.; Liu, C.; Shangguan, D.; Yang, W.; Gao, M. Protease-activated ratiometric fluorescent probe for $\mathrm{pH}$ mapping of malignant tumors. ACS Nano 2015, 9, 3199-3205. [CrossRef]

10. Bai, Z.; Chen, R.; Si, P.; Huang, Y.; Sun, H.; Kim, D.-H. Fluorescent pH sensor based on Ag@ SiO2 core-shell nanoparticle. ACS Appl. Mater. Interfaces 2013, 5, 5856-5860. [CrossRef]

11. Hong, S.W.; Jo, W.H. A fluorescence resonance energy transfer probe for sensing $\mathrm{pH}$ in aqueous solution. Polymer 2008, 49, 4180-4187. [CrossRef]

12. Rasheed, H.S.; Ahmed, N.M.; Matjafri, M. Ag metal mid layer based on new sensing multilayers structure extended gate field effect transistor (EG-FET) for pH sensor. Mater. Sci. Semicond. Process. 2018, 74, 51-56. [CrossRef]

13. Ang, P.K.; Chen, W.; Wee, A.T.S.; Loh, K.P. Solution-gated epitaxial graphene as pH sensor. J. Am. Chem. Soc. 2008, 130, 14392-14393. [CrossRef] [PubMed]

14. Cui, D.; Xie, C.; Pu, K. Development of semiconducting polymer nanoparticles for photoacoustic imaging. Macromol. Rapid Commun. 2017, 38, 1700125. [CrossRef] [PubMed]

15. Guo, L.; Ge, J.; Wang, P. Polymer dots as effective phototheranostic agents. Photochem. Photobiol. 2018, 94, 916-934. [CrossRef] [PubMed]

16. Wu, C.; Chiu, D.T. Highly fluorescent semiconducting polymer dots for biology and medicine. Angew. Chem. (Int. Ed. English) 2013, 52, 3086-3109. [CrossRef] [PubMed]

17. Liu, X.; Wang, T.; Wang, W.; Zhou, Z.; Yan, Y. A tailored molecular imprinting ratiometric fluorescent sensor based on red/blue carbon dots for ultrasensitive tetracycline detection. J. Ind. Eng. Chem. 2019, 72, 100-106. [CrossRef]

18. Wang, X.; Yu, S.; Liu, W.; Fu, L.; Wang, Y.; Li, J.; Chen, L. Molecular imprinting based hybrid ratiometric fluorescence sensor for the visual determination of bovine hemoglobin. ACS Sensors 2018, 3, 378-385. [CrossRef]

19. Jin, T.; Sasaki, A.; Kinjo, M.; Miyazaki, J. A quantum dot-based ratiometric pH sensor. Chem. Commun. 2010, 46, 2408-2410. [CrossRef]

20. Li, Y.; Wang, Y.; Yang, S.; Zhao, Y.; Yuan, L.; Zheng, J.; Yang, R. Hemicyanine-based high resolution ratiometric near-infrared fluorescent probe for monitoring $\mathrm{pH}$ changes in vivo. Anal. Chem. 2015, 87, 2495-2503. [CrossRef]

21. Sun, J.; Ling, P.; Gao, F. A Mitochondria-Targeted Ratiometric Biosensor for $\mathrm{pH}$ Monitoring and Imaging in Living Cells with Congo-Red-Functionalized Dual-Emission Semiconducting Polymer Dots. Anal. Chem. 2017, 89, 11703-11710. [CrossRef]

22. Chan, Y.-H.; Wu, C.; Ye, F.; Jin, Y.; Smith, P.B.; Chiu, D.T. Development of ultrabright semiconducting polymer dots for ratiometric $\mathrm{pH}$ sensing. Anal. Chem. 2011, 83, 1448-1455. [CrossRef] [PubMed]

23. Ma, L.Y.; Wang, H.Y.; Xie, H.; Xu, L.X. A long lifetime chemical sensor: study on fluorescence property of fluorescein isothiocyanate and preparation of $\mathrm{pH}$ chemical sensor. Spectrochim. Acta. Part. A Mol. Biomol. Spectrosc. 2004, 60, 1865-1872. [CrossRef] [PubMed]

Sample Availability: Sample Availability: Samples of the compounds in this article are not available from the authors.

(C) 2019 by the authors. Licensee MDPI, Basel, Switzerland. This article is an open access article distributed under the terms and conditions of the Creative Commons Attribution (CC BY) license (http://creativecommons.org/licenses/by/4.0/). 